\title{
SPECTRAL INDEX - REDSHIFT RELATION FOR RADIO GALAXIES AND QUASARS
}

\author{
R.D.DAGKESAMANSKII \\ Astrospace Center of P.N.Lebedev Physics Institute of RAS \\ Moscow Leniskii prospekt 53 Russia
}

Cosmological evolution of synchrotron spectra of the powerful extragalactic radio sources was studied by many authors. Some indications of such an evolution had been found firstly by analysis of 'spectral index - flux density' $(\alpha-S)$ relation for the sample of relatively strong radio sources $[1,2]$. Later Gopal-Krishna and Steppe [3] extended the analysis to weaker sources and found that the slope of $\alpha_{\text {med }}(S)$ curve changes dramatically at intermediate flux densities. Gopal-Krishna and Steppe pointed out that the maxima of the $\alpha_{m e d}(S)$ curve and of differential source counts are at almost the same flux density ranges (see [3], Fig.2). It has to be noticed that the all mentioned results were obtained using the low-frequency spectral indices and on the basis of low frequency samples.

It has been found also that the relation between the mean spectral indices of extragalactic radio sources and their flux densities is mainly due to $\alpha(S)$ relationship for corresponding quasar subsample [1]. For this reason a distribution of quasars on the 'spectral index - redshift' diagram was analysed and it was shown [4] that there were no the relatively flat spectrum $(\alpha>-0.7)$ sources in $3 \mathrm{C}$ catalogue which are identified with high redshift quasars $(z>1.0)$.

Recently, Herbig and Readhead [5] have published some results of their compilation of multi-frequency flux density measurements for three complete samples of strong extragalactic radio sources. The results were presented as tables of the redshifts, luminosities and spectral indices of the sources at three standard rest frequencies: $0.15,2.5$ and $40 \mathrm{GHz}$. We used this data to study the distribution on $(z, \alpha)$-plane of the sources from the well-known low-frequency complete sample defined by Laing, Riley and Longair [6]. The sample contains 173 sources and more than $98 \%$ of them are identified and have reliable redshifts. So, we can be sure that there is 
no any selection effect in the data except for limitations on flux density $\left(S_{178} \geq 10 J y\right)$ and galactic latitude $\left(b>10^{\circ}\right)$.

Deficit of the distant 'flat' spectrum $\left(\alpha_{0.15}>-0.7\right)$ quasars in the sample is well seen in their distribution on the $\left(z, \alpha_{0.154}\right)$-plane. This confirms the remarkable feature of quasar's $(\alpha-z)$ distribution found in [4] from different flux density measurements and for partly different samples of quasars. The same feature peeps out also at $\left(z-\alpha_{0.15}\right)$ diagram plotted for the subsample of radio galaxies.

Statistical tests applied to the both distributions show that the absence of the 'flat' spectrum objects at high redshifts is significant. A distribution similar to this could be expected if the 'flat' and 'steep' spectrum radio sources have very different luminosity functions. Indeed, if the 'flat' spectrum radio sources are intrinsically much weaker (at low frequencies) compared with the 'steep' spectrum objects then there will be only relatively near 'flat' spectrum objects in flux density limited samples.

However, 'flat' spectrum quasars median luminosity is only 2 times less than the 'steep' spectrum one's. On the other hand, we have found that $V / V_{\max }$ median values are very different for the groups of quasars. Indeed, for 12 'flat' spectrum quasars $\left(V / V_{\text {max }}\right)_{\text {med }}=0.33 \pm 0.06$ while for 30 'steep' spectrum quasars $(V / V-\max )_{\text {med }}=0.59 \pm 0.04$. The last value is close to the $\left(V / V_{\text {max }}\right)$ estimates for many other quasar samples and reflects the well-known fact that quasar's spatial density rises when the distance from observer increases. But $\left(V / V_{\text {max }}\right)_{\text {med }}$ for the 'flat' spectrum quasars is surprisingly low. Does this mean that their spatial density decrease with the distance from observer? If it is so, then these objects have to represent some separate group of extragalactic radio sources. This conclusion would have grave consequences and has to be checked carefully.

I would like to thank the Conference organizers for financial help which made me possible to attend this Conference. This work was supported as a part of Russian State Program "Astronomy" (via the SEC "Cosmion").

\section{References}

[1] Dagkesamanskii, R.D. (1969) Astrofizika,Vol. 5,pp 297-304

[2] Murdoch, H.S. (1976) Mon. Not. Roy.Astron. Soc.,Vol. 177,p 441

[3] Gopal-Krishna and Steppe, H. (1982) Astron.Astrophys., Vol. 113,pp 150-154

[4] Dagkesamanskii, R.D. (1970) Nature, Vol. 226,p 432

[5] Herbig, T. and Readhead, A.C.S. (1992) Astroph. J. Suppl.,Vol. 81,pp 83-124

[6] Laing, R.A., Riley, J.M., and Longair, M.S. (1983) Mon. Not. Roy. Astron. Soc., Vol. 204,p 151 